\title{
QTL analysis for early-maturing traits in cotton using two upland cotton (Gossypium hirsutum L.) crosses
}

\author{
Chengqi Li ${ }^{1}$, Xiaoyun Wang'), Na Dong1), Haihong Zhao'), Zhe Xia' ${ }^{2)}$, Rui Wang'), Richard L. Converse ${ }^{3)}$ \\ and Qinglian Wang*1) \\ 1) Henan Institute of Science and Technology, Key Discipline Open Lab on Crop Molecular Breeding of Henan Institute of Higher \\ Learning, Cotton Research Institute, Xinxiang Henan 453003, China \\ 2) College of Life Sciences, Henan Normal University, Xinxiang Henan 453003, China \\ 3) University of Cincinnati Blue Ash College, 9555 Plainfield Rd, Blue Ash, OH 45236, USA
}

\begin{abstract}
Making use of the markers linked closely to QTL for early-maturing traits for MAS (Marker-assisted selection) is an effective method for the simultaneous improvement of early maturity and other properties in cotton. In this study, two $\mathrm{F}_{2}$ populations and their $\mathrm{F}_{2: 3}$ families were generated from the two upland cotton (Gossypium hirsutum L.) crosses, Baimian $2 \times$ TM-1 and Baimian $2 \times$ CIR12. QTL for early-maturing traits were analyzed using $\mathrm{F}_{2: 3}$ families. A total of 54 QTL ( 31 suggestive and 23 significant) were detected. Fourteen significant QTL had the LOD scores not only $>3$ but also exceeding permutation threshold. At least four common QTL, $q B P-17$ for bud period (BP), $q G P-17 a / q G P-17 b$ ( $q G P-17$ ) for growth period (GP), $q Y P B F-$ $17 a / q Y P B F-17 b(q Y P B F-17)$ for yield percentage before frost (YPBF) and $q H F F B N-17$ for height of first fruiting branch node (HFFBN), were found in both populations. These common QTL should be reliable and could be used for MAS to facilitate early maturity. The common QTL, $q B P-17$, had a LOD score not only $>3$ but also exceeding permutation threshold, explaining $12.6 \%$ of the phenotypic variation. This QTL should be considered preferentially in MAS. Early-maturing traits of cotton are primarily controlled by dominant and over-dominant effects.
\end{abstract}

Key Words: short-season upland cotton, early-maturing traits, QTL, common QTL, MAS.

\section{Introduction}

Short-season upland cotton (Gossypium hirsutum L.), which is also called early-maturing cotton, generally exhibits a dwarf, compact phenotype with fewer leaves, shorter internodes and fruiting branches, and shorter growth period than middle-late-maturing cotton (Yu and Huang 1990). The selection and popularization of short-season cotton has significant value in alleviating the contradiction presented when occupying farmlands with either cotton or cereals, while optimizing cropping system. Early maturity of cotton is a comprehensive trait, encompassing growth period, individual growth stages, yield percentage before frost, first fruiting branch node and height of first fruiting branch node. These components that contribute to early maturity are all complex quantitative traits (White 1966), which are controlled by both QTL (quantitative trait locus/loci) and environment and manifest a variety of genetic modes in different combinations (Dong et al. 2010, Song et al. 2005). In addition, many researchers believe that a significant negative correlation ex-

Communicated by Duncan Vaughan

Received September 4, 2012. Accepted October 27, 2012.

*Corresponding author (e-mail: cottonmol@yahoo.cn) ists between early maturity and yield, and between early maturity and quality (Fan et al. 2006b, Song et al. 2005). Obtaining a satisfactory yield and quality in a short growing season is likely to exacerbate the conflict between early maturity and yield and between early maturity and quality, so it is increasingly difficult to improve upon these agriculturally desirable traits in short-season cotton simultaneously by means of traditional breeding.

Since the first elaborated RFLP map of allotetraploid cotton was published (Reinisch et al. 1994), genetic mapping in cotton, especially interspecific genetic mapping for Gossypium hirsutum $\times$ G. Barbadense, has made remarkable progress (Guo et al. 2007, Lacape et al. 2009, Nguyen et al. 2004). However, interspecific genetic maps are hard to apply directly to the genetic improvement of upland cotton (Gossypium hirsutum L.). At present, intraspecific genetic maps in cotton are relatively less advanced because of limited genetic diversity and low polymorphism (Shappley et al. 1998, Shen et al. 2005, Ulloa et al. 2002, Zhang et al. 2009). To take advantage of markers linked closely to target genes for marker-assisted selection (MAS), a highly saturated intraspecific genetic map in upland cotton covering the entire genome is a necessity. Yu et al. (2010) constructed a comprehensive reference map that contained 7,424 markers 
and represented over $93 \%$ of the combined information from 28 individual maps, which laid an important foundation for exploration and utilization of cotton gene resources.

Construction of a genetic linkage map in short-season upland cotton and identification of QTL for early-maturing traits, as well as making use of the markers linked closely to target genes for MAS, is an effective method for the simultaneous improvement of early maturity and other properties. The first genetic linkage map in short-season upland cotton was constructed by using a $F_{2}$ population obtained from the cross between the short-season upland cotton cultivar, CIR36 and the genetic standard line of Gossypium hirsutum L., TM-1 (Fan et al. 2006a). The map contained five linkage groups and 43 markers, representing a total length of $1,174 \mathrm{cM}$ covering $23.48 \%$ of the cotton genome, with 12 QTL for early-maturing traits detected. Guo et al. (2008) reported another genetic map that contained 70 molecular markers and three morphological markers distributed in 17 linkage groups covering $650.8 \mathrm{cM}$, approximately $14.5 \%$ of the cotton genome. Ultimately, five QTL for first fruiting branch node were detected. Zhang et al. (2008) used a recombinant inbred line (RIL) population raised from the cross between the upland cotton Acala 1517 (ZM-06339) and Dezhou 047 to construct a genetic map; 51 SSR markers were assigned to 15 linkage groups, about $504.05 \mathrm{cM}$ (10.08\% of the cotton genome) was covered and three significant QTL for growth period were detected.

From above studies, it can be concluded that only preliminary progress of the genetic map construction in shortseason upland cotton has been made; few QTL for earlymaturing traits have been identified and their reliability has yet to receive further verification. In this study, the shortseason upland cotton cultivar, Baimian2, was used as the common parent to cross respectively with TM-1 and CIR12 and two $F_{2}$ populations and their $F_{2: 3}$ families were generated. $\mathrm{F}_{2: 3}$ families were used to analyze QTL for earlymaturing traits based on the maps constructed from the $F_{2}$ populations. Our objective was to provide a theoretical basis for the genetic structure analysis and marker-assisted selection for early maturity of cotton.

\section{Materials and Methods}

\section{Mapping population}

Three upland cotton cultivars of varying maturity were chosen as the parents to generate two mapping populations: $\mathrm{F}_{2}$ population (Pop I) and its $\mathrm{F}_{2: 3}$ families raised from Baimian $2 \times$ TM-1 and $F_{2}$ population (Pop II) and its $F_{2: 3}$ families raised from Baimian2 $\times$ CIR12. Baimian2 is a Chinese commercial short-season cotton bred by Henan Institute of Science and Technology using the pedigree method and characterized by the desirable features of early maturity, high quality, disease resistance and wide adaptability (Zhu et al. 2008). TM-1 is a genetic standard line of upland cotton in the United States and exhibits a latematuring phenotype in the Yangtze River basin, Yellow
River basin and northwestern inland of China (Ai et al. 2010, Kohel et al. 1970, Li et al. 2011). CIR12 is a Chinese commercial multiple-hybrid line bred by Cotton Research Institute of Chinese Agricultural Academy exhibiting a middle-maturing phenotype in China (Tan and Liu 1990). In the summer of 2008, Baimian2 was crossed with both TM-1 and CIR12 to obtain $F_{1}$ seeds in the Henan Institute of Science and Technology experimental field. In the winter of $2008, F_{1}$ individual plants were self-pollinated and $F_{2}$ seeds were harvested in Hainan Province of China. In 2009, $\mathrm{F}_{2}$ individual plants were self-pollinated and $\mathrm{F}_{2: 3}$ family seeds were harvested; in 2010, parents and $\mathrm{F}_{2: 3}$ families (numbering 220 and 208, respectively) for the two $F_{2}$ populations were grown in a single-row plot ( $0.8 \mathrm{~m}$ wide, $5 \mathrm{~m}$ long) with two replicates and a random complete block design.

\section{Phenotypic data collection and statistical analysis}

To reduce the environmental error, the average value of every $\mathrm{F}_{2: 3}$ family row was used as the estimated value of the corresponding $\mathrm{F}_{2}$ individual plant. Seven early-maturing traits of both $\mathrm{F}_{2: 3}$ families were investigated. These traits comprised: seedling period (SP; the period from seedling emergence to flower bud); bud period (BP; the period from flower bud emergence to flowering); flower and boll period (FBP; the period from flowering to boll opening); growth period (GP; the period from seedling emergence to boll opening); yield percentage before frost (YPBF); first fruiting branch node (FFBN; the node from cotyledon node to first fruiting branch node) and height of first fruiting branch node (HFFBN; the distance between cotyledon node and NFFB). Ten plants of each family row were investigated and the average of two replicates was recorded as the last phenotypic value. The phenotypic data were analyzed using statistical software SPSS 17.0 (SPSS, Chicago, USA).

\section{DNA marker Assays}

Genomic DNA of individual plants of both $F_{2}$ populations was extracted using a modified CTAB method (Paterson et al. 1993). A total of 4,083 SSR primers were used to screen for polymorphism. These primers including the series of BNL, CER, CGR, CIR, CM, COT, DPL, DC, GH, HAU, JESPR, MUCS, MUSB, MUSS, MGHES, NAU, SHIN, STV and TMB, were mainly selected from the published cotton interspecific and intraspecific maps (Guo et al. 2007, Nguyen et al. 2004, Qin et al. 2008, Zhang et al. 2012), as well as reported markers linked to QTL for agriculturally significant traits of cotton (Jiang et al. 2009, Lacape et al. 2005, Li et al. 2008, Mei et al. 2004, Qin et al. 2008, Zhang et al. 2003, Zhang et al. 2012). The primer sequences were obtained from the Cotton Marker Database (http://www.cottonmarker.org) and synthesized by Nanjing Jinsirui Biology Engineering Co., Ltd. The protocol for PCR amplification and examination followed that of Zhang et al. (2002). A chi-square test was performed to determine if the allele frequency at each individual marker locus segregated normally. 


\section{Map construction and QTL detection}

Map construction were performed for the two $\mathrm{F}_{2}$ populations by JoinMap 3.0 (Van Ooijen and Voorrips 2001) with a LOD score of 3.0 and a recombination frequency of 0.50 . $\mathrm{F}_{2: 3}$ families for Pop I and Pop II were used to identify QTL. QTL were detected by composite interval mapping (Zeng 1994) using Windows QTL Cartographer 2.5 (Wang et al. 2006). The QTL with a LOD value between 2.0 and 3.0 was defined as a suggestive QTL (Lander and Kruglyak 1995) and the QTL with a LOD value $\geq 3.0$ (Jiang et al. 1998), or $\geq$ the threshold value calculated by a permutation test with 1000 times, was defined as a significant QTL (Churchill and Doerge 1994). QTL confidence intervals (90\% and 95\%) were set as map intervals corresponding to one and two LOD declines on either side of the peak. The absolute value of the ratio of dominant effect to additive effect (D/A) was used to classify the gene action mode. If the absolute value was $\leq 1$, it was regarded as additive effect (A), if the absolute value $>1$, it was regarded as dominant effect (D); if $>3$, it was regarded as over-dominant effect (OD) (Paterson et al. 2003).

QTL nomenclature followed the protocol used in rice (McCouch et al. 1997). The QTL name begin with ' $q$ ', followed by an abbreviation of the trait, then the name of the Chr. (subgenome) or linkage group. If there was more than one QTL for the same trait on a linkage group, lowercase letters were used to distinguish them. Maps were drawn using MapChart 2.2 (Voorrips 2006). Linkage groups were localized to Chr. (subgenomes) by using the markers that had been previously anchored (Guo et al. 2007, Han et al. 2006, Mei et al. 2004). LGX was used to designate linkage groups not localized to Chr. (subgenomes).

\section{Results}

Performance of early-maturing traits for parents and $F_{2: 3}$ families

Statistical analysis of early-maturing traits for parents and $\mathrm{F}_{2: 3}$ populations are listed in Table 1 . The differences in all the seven early-maturing traits were significant or highly significant between the parents of both populations, suggesting that the parents we selected are ideal to search for the genes responsible for early maturity. In Pop I, the difference in first fruiting branch node (FFBN) was significant between Baimian 2 and TM-1; in Pop II, the difference in seedling period (SP) was significant between Baimian2 and CIR12; the differences in all the other early-maturing traits were highly significant in the two populations. Statistical analysis of early-maturing traits for $\mathrm{F}_{2: 3}$ families showed that most of the traits for both $\mathrm{F}_{2: 3}$ families displayed transgressive segregation; of all traits, the standard deviation (SD) of growth period (GP) was at a maximum (3.39 and 3.13) and that of yield percentage before frost (YPBF) was at a minimum ( 0.04 and $0.05)$; skewness of all traits was $<1$, which is consistent with the normal distribution characteristics of quantitative traits.

\section{Correlation among early-maturing traits}

Correlation analysis among early-maturing traits is given in Table 2. Correlations among most traits showed high consistency in $\mathrm{F}_{2: 3}$ families of both populations. To illustrate, seedling period (SP) was significant or highly significant positively correlated with bud period (BP), flower and boll period (FBP) and growth period (GP) and was significant or highly significant negative correlated with yield percentage before frost (YPBF) and was non-significant positively correlated with first fruiting branch node (FFBN). Obviously, correlations among some traits showed differences in $\mathrm{F}_{2: 3}$ families of both populations. Notably, the correlations between yield percentage before frost (YPBF) and first fruiting branch node (FFBN) and between yield percentage before frost (YPBF) and height of first fruiting branch node (HFFBN) displayed non-significant negative correlations in $\mathrm{F}_{2: 3}$ families of Pop I, but the correlation between them was non-significant positive and highly significant negative in $\mathrm{F}_{2: 3}$ families of Pop II, respectively.

\section{Genetic map construction}

A total of 295 and 169 polymorphic marker loci from the 4,083 SSR primers were obtained for Pop I and Pop II,

Table 1. Statistical analysis of early-maturing traits for parents and $F_{2: 3}$ families

\begin{tabular}{|c|c|c|c|c|c|c|c|c|c|c|c|c|c|c|}
\hline \multirow{3}{*}{ Trait } & \multicolumn{7}{|c|}{ Pop I/Baimian2 $\left(\mathrm{P}_{2}\right) \times$ TM-1 $\left(\mathrm{P}_{1}\right)$} & \multicolumn{7}{|c|}{ Pop II/Baimian2 $\left(\mathrm{P}_{2}\right) \times \operatorname{CIR} 12\left(\mathrm{P}_{1}\right)$} \\
\hline & \multicolumn{3}{|c|}{ Parents } & \multicolumn{4}{|c|}{$\mathrm{F}_{2: 3}$ family } & \multicolumn{3}{|c|}{ Parents } & \multicolumn{4}{|c|}{$\mathrm{F}_{2: 3}$ family } \\
\hline & $\mathrm{P}_{1}$ & $\mathrm{P}_{2}$ & $\mathrm{P}_{1}-\mathrm{P}_{2}$ & Range & Mean & $\mathrm{SD}$ & Skewness & $\mathrm{P}_{1}$ & $\mathrm{P}_{2}$ & $\mathrm{P}_{1}-\mathrm{P}_{2}$ & Range & Mean & $\mathrm{SD}$ & Skewness \\
\hline $\mathrm{SP}$ (d) & 37.71 & 35.07 & $2.64 * *$ & $34.38-38.11$ & 36.08 & 0.51 & 0.79 & 35.50 & 34.82 & $0.68 *$ & $35.00-37.36$ & 35.94 & 0.36 & 0.95 \\
\hline $\mathrm{BP}(\mathrm{d})$ & 23.14 & 19.28 & $3.86^{* *}$ & $18.69-24.91$ & 22.12 & 1.17 & -0.22 & 22.71 & 19.27 & $3.44 * *$ & $17.77-23.71$ & 20.92 & 1.06 & 0.02 \\
\hline FBP (d) & 49.71 & 39.79 & $9.92 * *$ & $41.00-50.18$ & 46.29 & 2.25 & 0.59 & 47.30 & 43.09 & $4.21 * *$ & $39.58-50.08$ & 44.21 & 2.15 & 0.23 \\
\hline GP (d) & 110.57 & 93.86 & $16.71^{* *}$ & $96.00-115.82$ & 104.55 & 3.39 & 0.19 & 105.60 & 97.18 & $8.42 * *$ & $94.23-109.86$ & 101.11 & 3.13 & 0.19 \\
\hline YPBF & 0.61 & 1.00 & $-0.39 * *$ & $0.83-1.00$ & 0.96 & 0.04 & -0.98 & 0.89 & 1.00 & $-0.11 * *$ & $0.76-1.00$ & 0.95 & 0.05 & -0.92 \\
\hline FFBN & 5.67 & 5.00 & $0.67 *$ & $4.20-6.80$ & 5.59 & 0.53 & -0.3 & 4.78 & 3.70 & $1.08 * *$ & $3.30-7.40$ & 5.25 & 0.94 & 0.19 \\
\hline $\begin{array}{l}\text { HFFBN } \\
(\mathrm{cm})\end{array}$ & 13.80 & 8.76 & $5.04 * *$ & $8.47-18.02$ & 12.22 & 1.71 & 0.35 & 18.91 & 10.50 & $8.41^{* *}$ & $11.00-19.60$ & 14.63 & 1.8 & 0.44 \\
\hline
\end{tabular}

$\mathrm{SP}=$ seedling period, $\mathrm{BP}=$ bud period, $\mathrm{FBP}=$ flower and boll period, $\mathrm{GP}=$ growth period, $\mathrm{YPBF}=\mathrm{yield}$ percentage before frost, $\mathrm{FFBN}=$ first fruiting branch node, HFFBN = height of first fruiting branch node.

$*, * *$ Indicate significance at 0.05 and 0.01 levels, respectively. 
Table 2. Correlation analyses among early-maturing traits

\begin{tabular}{lccccccc}
\hline \hline Trait & SP & BP & FBP & GP & YPBF & FFBN & HFFBN \\
\hline SP & & $0.466^{* *}$ & $0.289^{* *}$ & $0.520^{* *}$ & $-0.230^{* *}$ & 0.046 & 0.137 \\
BP & $0.595^{* *}$ & & $0.512^{* *}$ & $0.776^{* *}$ & $-0.390^{* *}$ & $0.264^{* *}$ & $0.334^{* *}$ \\
FBP & $0.272^{* *}$ & $0.533^{* *}$ & & $0.893^{* *}$ & $-0.313^{* *}$ & $0.153^{*}$ & $0.352^{* *}$ \\
GP & $0.567^{* *}$ & $0.818^{* *}$ & $0.892^{* *}$ & & $-0.397^{* *}$ & $0.210^{* *}$ & $0.383^{* *}$ \\
YPBF & $-0.246^{* *}$ & $-0.305^{* *}$ & $-0.423^{* *}$ & $-0.432^{* *}$ & & -0.129 & -0.115 \\
FFBN & 0.035 & $0.200^{* *}$ & 0.058 & 0.123 & 0.004 & $0.512^{* *}$ \\
HFFBN & $0.173^{*}$ & $0.325^{* *}$ & $0.358^{* *}$ & $0.380^{* *}$ & $-0.303^{* *}$ & $0.302^{* *}$ & \\
\hline
\end{tabular}

Correlation coefficients among early-maturing traits in $\mathrm{F}_{2: 3}$ family of Pop I above the diagonal and in $\mathrm{F}_{2: 3}$ family of Pop II under the diagonal. *,** Indicate significance at 0.05 and 0.01 levels, respectively.

See Table 1 for abbreviations.
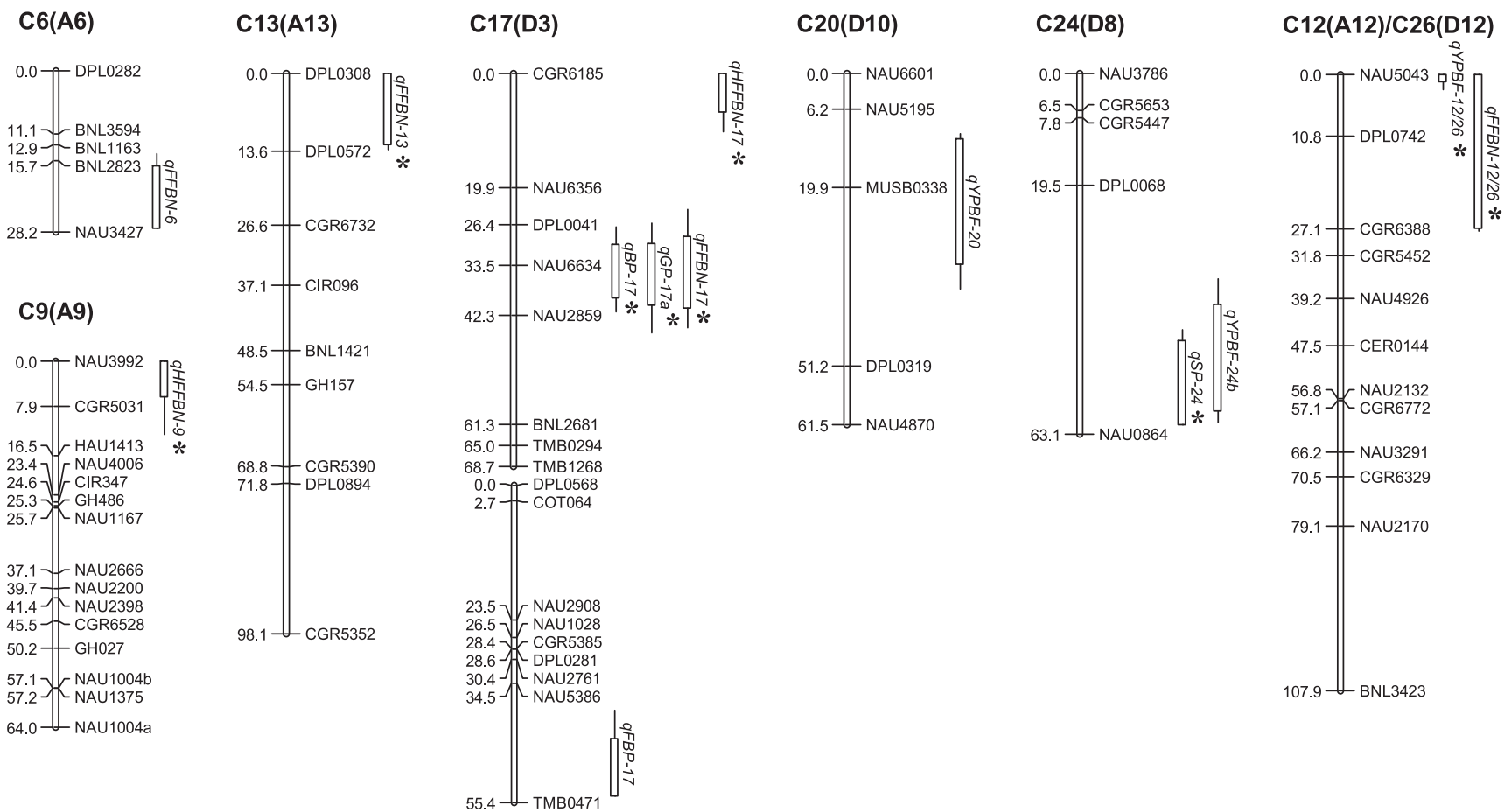

Fig. 1. Location of significant QTL for early-maturing traits in Pop I (Baimian2 $\times$ TM-1). 13 significant QTL are shown as seedling period (SP), bud period (BP), flower and boll period (FBP), growth period (GP), yield percentage before frost (YPBF), first fruiting branch node (FFBN) and height of first fruiting branch node (HFFBN). * indicates the QTL had a LOD score not only $>3$ but also exceeding permutation threshold.

respectively. A chi-square test indicated that eight and 10 polymorphic loci displayed a segregation distortion (data not shown). All polymorphic loci were used to construct the genetic map. Map I (Pop I) included 269 loci localized into 43 linkage groups that span $1,837.8 \mathrm{cM}$ (approximately $36.76 \%$ of the cotton genome) with an average distance of $6.8 \mathrm{cM}$ between markers. Map II (Pop II) included 127 loci localized into 33 linkage groups that span $1,244.3 \mathrm{cM}$ (approximately $24.89 \%$ of the cotton genome) with an average distance of $9.7 \mathrm{cM}$ between markers. Taking into account the objective of this study, only those linkage groups associated with the detected QTL were presented.

\section{QTL analysis}

A total of 54 QTL, with 31 suggestive and 23 significant, for early-maturing traits were detected (Table 3 ). They were distributed in $24 \mathrm{Chr}$. (subgenomes) or linkage groups (Figs. 1, 2, only significant QTL were displayed). Fourteen significant QTL had the LOD scores not only $>3$ but also exceeding permutation threshold. They might truly exist in cotton genome.

Seedling period (SP): Six QTL for SP were detected in six Chr. (subgenomes) or linkage groups. In Pop I, one suggestive QTL and one significant QTL were detected in C6 (A6) and C24 (D8), explaining 5.4-42.0\% of the phenotypic variation; the significant QTL $q S P-24$ had a LOD score not only $>3$ but also exceeding permutation threshold. In Pop II, two suggestive and two significant QTL were detected in C8 (A8), C14 (A14), LG5 and LG6, explaining 5.8-46.3\% of the phenotypic variation; the significant QTL $q S P-L G 5$ had a LOD score not only $>3$ but also exceeding permutation threshold. Among these six QTL, two represented additive 
158

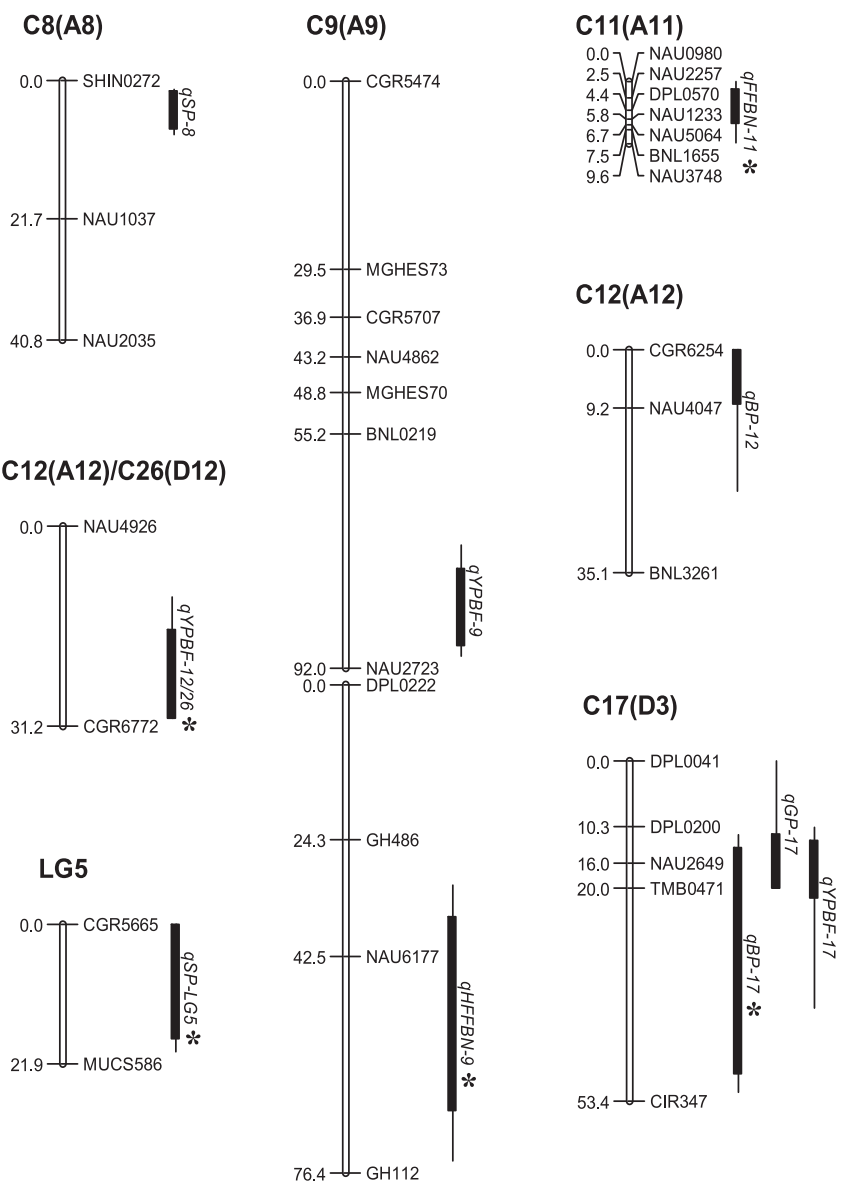

Fig. 2. Location of significant QTL for early-maturing traits from Pop II (Baimian $2 \times$ CIR12). Ten significant QTL are shown as seedling period (SP), bud period (BP), growth period (GP), yield percentage before frost (YPBF), first fruiting branch node (FFBN) and height of first fruiting branch node (HFFBN). * indicates that the QTL had a LOD score not only $>3$ but also exceeding permutation threshold.

effects and four represented dominant effects; decreased seedling period was conferred by Baimian 2 allele in all instances.

Bud period (BP): Seven QTL for BP were detected in six Chr. (subgenomes) or linkage groups. In Pop I, four suggestive and one significant QTL were detected in C1 (A1), C10 (A10), C17 (A17), C12 (A12)/26 (D12) and LG2, explaining $4.6-12.6 \%$ of the phenotypic variation; the significant QTL $q B P-17$ had a LOD score not only $>3$ but also exceeding permutation threshold. In Pop II, two significant QTL was detected in $\mathrm{C} 12$ (A12), C17 (D3), explaining 6.5-12.6\% of the phenotypic variation; the significant QTL $q B P-17$ had a LOD score not only $>3$ but also exceeding permutation threshold. Among these seven QTL, four represented additive effects and three represented dominant or overdominant effects; decreased bud period was conferred by Baimian2 allele at six QTL and by CIR12 allele at one QTL.

Flower and boll period (FBP): Eight QTL for FBP were detected in eight $\mathrm{Chr}$. (subgenomes) or linkage groups. In Pop I, four suggestive and one significant QTL were detect- ed in C9 (A9), C16 (D7), C17 (D3), C22 (D4) and LG2, explaining $4.7-14.6 \%$ of the phenotypic variation. In Pop II, three suggestive QTL were detected in C3 (A3), C7 (A7), C8 (A8)/C24 (D8), explaining $6.5-12.1 \%$ of the phenotypic variation. Among these eight QTL, five represented additive effects while three represented dominant or over-dominant effects; decreased flower and boll period was conferred by Baimian 2 allele at 6 QTL and by TM-1 or CIR 12 allele at 2 QTL.

Growth period (GP): Five QTL were detected for GP on three Chr. (subgenomes) or linkage groups. In Pop I, three suggestive and one significant QTL were detected on C13 (A13), C17 (D3) and C24 (D8), explaining 5.7-13.8\% of the phenotypic variation. Notably, the significant QTL $q G P-17 a$ had a LOD score not only $>3$ but also exceeding permutation threshold. In Pop II, one significant QTL was detected on $\mathrm{C} 17$ (D3), explaining $20.0 \%$ of the phenotypic variation. Among these five QTL, two represented additive effects and three represented dominant or over-dominant effects; decreased growth period was entirely conferred by Baimian2 allele.

Yield percentage before frost $(Y P B F)$ : Fourteen QTL for YPBF were detected in nine Chr. (subgenomes) or linkage groups. In Pop I, five suggestive and three significant QTL were detected on C10 (A10), C11 (A11), C17 (D3), C20 (D10), C24 (D8) and C12 (A12)/26 (D12) and explaining $5.3-46.7 \%$ of the phenotypic variation. It should be noted that the significant QTL $q Y P B F-12 / 26$ had a LOD score not only $>3$ but also exceeding permutation threshold. In Pop II, three suggestive and three significant QTL were detected in C9 (A9), C17 (D3), C12 (A12)/26 (D12), LG5 and LG6, explaining $8.0-42.3 \%$ of the phenotypic variation. Note that the significant QTL $q Y P B F-12 / 26$ had a LOD score not only $>3$ but also exceeding permutation threshold. Among these 14 QTL, six represented additive effects and eight represented dominant or over-dominant effects; increased yield percentage before frost was conferred by Baimian2 allele at 13 QTL and by CIR12 allele at one QTL.

First fruiting branch node (FFBN): Eight QTL were detected for FFBN on eight Chr. (subgenomes) or linkage groups. In Pop I, two suggestive and four significant QTL were detected in C1 (A1), C6 (A6), C9 (A9), C13 (A13), $\mathrm{C} 17$ (D3) and C12 (A12)/26 (D12), explaining 4.7-19.1\% of the phenotypic variation. Here we note that the significant QTL $q F F B N-13, q F F B N-17$ and $q F F B N-12 / 26$ had the LOD scores not only $>3$ but also exceeding permutation threshold. In Pop II, one suggestive and one significant QTL were detected in C5 (A5), C11 (A11), explaining 6.1-7.5\% of the phenotypic variation. Notably, the significant QTL $q F F B N-11$ had a LOD score not only $>3$ but also exceeding permutation threshold. Among these eight QTL, five represented additive effects and three represented dominant or over-dominant effects; decreased first fruiting branch node was conferred by Baimian2 allele at six QTL and by CIR12 allele at two QTL.

Height of first fruiting branch node (HFFBN): Six QTL 
Table 3. Characteristics of QTL for early-maturing traits detected in both populations

\begin{tabular}{|c|c|c|c|c|c|c|c|c|c|c|c|c|c|}
\hline Trait & QTL & Pop & $\begin{array}{l}\text { Chr. (Subgenome) } \\
\text { or linkage group }\end{array}$ & $\begin{array}{l}\text { Position } \\
(\mathrm{cM})\end{array}$ & $\begin{array}{l}\text { Nearest } \\
\text { marker }\end{array}$ & LOD & $\begin{array}{l}\text { Permuta- } \\
\text { tion } \\
\text { threshold }\end{array}$ & $\mathrm{A}^{a}$ & $\mathrm{D}^{b}$ & $\mathrm{D} / \mathrm{A}$ & Mode & $R^{2}(\%)^{c}$ & $\begin{array}{l}\text { Favorable } \\
\text { gene }^{d}\end{array}$ \\
\hline \multirow[t]{6}{*}{ SP } & $q S P-6$ & I & C6(A6) & 15.7 & BNL2823 & 2.08 & 3.28 & -0.16 & -0.15 & 0.99 & A & 5.4 & Baimian2 \\
\hline & $q S P-24$ & I & C24(D8) & 55.1 & NAU0864 & $3.74^{*}$ & 3.28 & -0.56 & -0.64 & 1.14 & D & 42.0 & Baimian2 \\
\hline & $q S P-8$ & II & C8(A8) & 4.1 & SHIN0272 & 4.91 & 7.27 & -0.60 & -0.67 & 1.12 & D & 46.3 & Baimian2 \\
\hline & $q S P-14$ & II & C14(D2) & 0.1 & SHIN1339 & 2.37 & 7.27 & -0.10 & 0.17 & -1.75 & D & 5.8 & Baimian2 \\
\hline & $q S P-L G 5$ & II & LG5 & 2.1 & CGR5665 & $10.16^{*}$ & 7.27 & -0.82 & -0.71 & 0.87 & A & 41.9 & Baimian2 \\
\hline & $q S P-L G 6$ & II & LG6 & 19.8 & MUCS127 & 2.05 & 7.27 & -0.26 & -0.32 & 1.23 & D & 26.8 & Baimian2 \\
\hline \multirow[t]{7}{*}{ BP } & $q B P-1$ & I & C1(A1) & 73.7 & BNL2827 & 2.11 & 2.66 & -0.34 & -0.09 & 0.26 & A & 4.6 & Baimian2 \\
\hline & $q B P-10$ & I & C10(A10) & 0.1 & NAU2935 & 2.63 & 2.66 & 0.25 & 0.36 & 1.48 & $\mathrm{D}$ & 5.4 & TM-1 \\
\hline & $q B P-17$ & I & C17(D3) & 33.6 & NAU6634 & $5.68^{*}$ & 2.66 & -0.58 & -0.03 & 0.04 & A & 12.6 & Baimian2 \\
\hline & $q B P-12 / 26$ & I & $\mathrm{C} 12(\mathrm{~A} 12) / \mathrm{C} 26(\mathrm{D} 12)$ & 70.6 & CGR6329 & 2.22 & 2.66 & -0.18 & 0.45 & -2.48 & $\mathrm{D}$ & 4.9 & Baimian2 \\
\hline & $q B P-L G 2$ & I & LG2 & 1.1 & NAU2811 & 2.08 & 2.66 & -0.78 & 0.13 & -0.16 & A & 17.1 & Baimian2 \\
\hline & $q B P-12$ & II & C12(A12) & 0.1 & CGR6254 & 2.43 & 2.38 & -0.10 & 0.53 & -5.13 & OD & 6.5 & Baimian2 \\
\hline & $q B P-17$ & II & C17(D3) & 16.2 & NAU2649 & $5.21^{*}$ & 2.38 & -0.51 & -0.17 & 0.34 & A & 12.6 & Baimian2 \\
\hline \multirow[t]{8}{*}{ FBP } & $q F B P-9$ & I & C9(A9) & 63.3 & NAU1004a & 2.56 & 2.64 & -0.73 & -0.40 & 0.54 & A & 6.5 & Baimian2 \\
\hline & $q F B P-16$ & I & C16(D7) & 82.5 & NAU6078 & 2.02 & 2.64 & -0.31 & 0.86 & -2.82 & D & 4.7 & Baimian2 \\
\hline & $q F B P-17$ & I & C17(D3) & 54.2 & TMB0471 & 2.78 & 2.64 & -0.52 & -0.89 & 1.70 & D & 8.2 & Baimian2 \\
\hline & $q F B P-22$ & I & C22(D4) & 8.1 & NAU5046 & 2.56 & 2.64 & 0.60 & -0.81 & -1.36 & $\mathrm{D}$ & 6.8 & TM-1 \\
\hline & $q F B P-L G 2$ & I & LG2 & 21.9 & BNL1044 & 2.44 & 2.64 & -0.90 & -1.04 & 1.16 & D & 14.6 & Baimian2 \\
\hline & $q F B P-3$ & II & $\mathrm{C} 3(\mathrm{~A} 3)$ & 0.1 & DPL0209 & 2.03 & 2.41 & 0.52 & -1.03 & -1.99 & D & 7.5 & CIR12 \\
\hline & $q F B P-7$ & II & C7(A7) & 0.1 & NAU1043 & 2.11 & 2.41 & -0.32 & 1.03 & -3.27 & OD & 6.5 & Baimian2 \\
\hline & $q F B P-8 / 24$ & II & $\mathrm{C} 8(\mathrm{~A} 8) / \mathrm{C}$ & 3.1 & CGR5202 & 2.22 & & -1.11 & -0.72 & 0.65 & A & 12.1 & Baimian2 \\
\hline \multirow[t]{5}{*}{ GP } & $q G P-13$ & I & C13(A & 0 . & CIR096 & 2.0 & & -0.07 & -1.58 & 22.54 & OD & 5.7 & Baimian2 \\
\hline & $q G P-17 a$ & I & C17(I & 35.5 & NAU6634 & $4.84^{*}$ & & -1.74 & 0.20 & -0.11 & A & 13.8 & Baimian2 \\
\hline & $q G P-17 b$ & I & $\mathrm{C} 17($ & 50.9 & TMB04 & 2.34 & & -0.80 & -1.59 & 2.00 & D & 9.5 & Baimian2 \\
\hline & $q G P-24$ & I & $\mathrm{C} 21$ & 8.1 & CGR & & & - & -0.69 & 0.71 & A & 5.7 & Baimian2 \\
\hline & $q G P-17$ & II & & & & & & _ & -1.96 & 1.39 & $\mathrm{D}$ & 20.0 & Baimian2 \\
\hline \multirow[t]{14}{*}{ YPBF } & $q Y P B F-10$ & I & C10(A & & NAU0S & & 4.6 & -0.02 & -0.01 & 0.31 & A & 7.4 & Baimian2 \\
\hline & $q Y P B F-11$ & I & C11(A11) & & NAU5064 & 2.08 & 4.6 & -0.01 & 0.01 & -0.38 & A & 5.3 & Baimian2 \\
\hline & $q Y P B F-17 a$ & I & $\mathrm{C} 17$ & 39.6 & NAL & 2.73 & 4. & 0.02 & 0.02 & 0.75 & A & 10.9 & Baimian2 \\
\hline & $q Y P B F-17 b$ & I & $\mathrm{C} 17$ & 38.5 & NAL & 2.19 & 4.63 & 0.01 & -0.20 & -21.78 & OD & 7.2 & Baimian2 \\
\hline & $q Y P B F-20$ & I & $\mathrm{C} 20($ & 22.7 & MUS & 3.09 & 4.63 & 0.00 & 0.03 & 270.00 & OD & 10.9 & Baimian2 \\
\hline & $q Y P B F-24 a$ & I & C24(D8) & 10.3 & TMB0 & 2.37 & 4.63 & 0.01 & -0.01 & -0.69 & A & 6.2 & Baimian2 \\
\hline & $q Y P B F-24 b$ & I & C24(D8) & 49.6 & NAU0864 & 3.79 & 4.63 & 0.03 & 0.05 & 1.96 & $\mathrm{D}$ & 46.7 & Baimian2 \\
\hline & $q Y P B F-12 / 26$ & I & $\mathrm{C} 12(\mathrm{~A} 12) / \mathrm{C} 26(\mathrm{D} 12)$ & 0.1 & NAU5043 & $10.60^{*}$ & 4.63 & 0.04 & 0.04 & 1.13 & $\mathrm{D}$ & 9.0 & Baimian2 \\
\hline & $q Y P B F-9 a$ & II & C9(A9) & 3.3 & CGR5474 & 2.08 & 4.85 & 0.01 & 0.02 & 1.50 & $\mathrm{D}$ & 7.0 & Baimian2 \\
\hline & $q Y P B F-9 b$ & II & C9(A9) & 83.1 & NAU2723 & 3.87 & 4.85 & 0.05 & 0.04 & 0.71 & A & 40.7 & Baimian2 \\
\hline & $q Y P B F-17$ & II & C17(D3) & 16.1 & NAU2649 & 3.34 & 4.85 & 0.02 & 0.00 & 0.06 & A & 8.0 & Baimian2 \\
\hline & qYPBF-12/26 & II & $\mathrm{C} 12(\mathrm{~A} 12) / \mathrm{C} 26(\mathrm{D} 12)$ & 27.5 & CGR6772 & $5.11^{*}$ & 4.85 & 0.02 & 0.02 & 1.60 & $\mathrm{D}$ & 12.1 & Baimian2 \\
\hline & $q Y P B F-L G 5$ & II & LG5 & 0.1 & CGR5665 & 2.68 & 4.85 & 0.01 & 0.08 & 5.92 & OD & 42.3 & Baimian2 \\
\hline & $q Y P B F-L G 6$ & II & LG6 & 29.9 & MUCS127 & 2.03 & 4.85 & -0.02 & 0.04 & -2.00 & $\mathrm{D}$ & 19.6 & CIR12 \\
\hline \multirow[t]{8}{*}{ FFBN } & $q F F B N-1$ & I & $\mathrm{C} 1(\mathrm{~A} 1)$ & 23.6 & NAU5411 & 2.50 & 2.70 & 0.13 & -0.07 & -0.56 & A & 4.7 & TM-1 \\
\hline & $q F F B N-6$ & I & C6(A6) & 24.2 & NAU3427 & 2.77 & 2.70 & 0.17 & -0.25 & -1.41 & $\mathrm{D}$ & 8.6 & TM-1 \\
\hline & $q F F B N-9$ & I & C9(A9) & 11.9 & CGR5031 & 2.52 & 2.70 & -0.01 & -0.35 & 44.13 & OD & 12.0 & Baimian2 \\
\hline & $q F F B N-13$ & I & C13(A13) & 0.1 & DPL0308 & $3.17^{*}$ & 2.70 & -0.15 & -0.19 & 1.23 & $\mathrm{D}$ & 7.2 & Baimian2 \\
\hline & $q F F B N-17$ & I & C17(D3) & 34.7 & NAU6634 & $6 . .83^{*}$ & 2.70 & -0.34 & -0.09 & 0.26 & A & 19.1 & Baimian2 \\
\hline & $q F F B N-12 / 26$ & I & $\mathrm{C} 12(\mathrm{~A} 12) / \mathrm{C} 26(\mathrm{D} 12)$ & 12.8 & DPL0742 & $4.33^{*}$ & 2.70 & -0.24 & -0.08 & 0.33 & A & 10.6 & Baimian2 \\
\hline & $q F F B N-5$ & II & C5(A5) & 37.5 & DPL0417 & 2.27 & 2.94 & -0.35 & 0.25 & -0.73 & A & 6.1 & Baimian2 \\
\hline & $q F F B N-11$ & II & C11(A11) & 4.4 & DPL0570 & $3.11^{*}$ & 2.94 & -0.33 & -0.18 & 0.53 & A & 7.5 & Baimian2 \\
\hline \multirow[t]{6}{*}{ HFFBN } & $q H F F B N-9$ & I & С9(A9) & 0.1 & NAU3992 & $3.02 *$ & 2.83 & -0.46 & -0.59 & 1.28 & D & 7.1 & Baimian2 \\
\hline & $q H F F B N-17$ & I & C17(D3) & 0.1 & CGR6185 & $5.38^{*}$ & 2.83 & -0.89 & -0.40 & 0.46 & A & 13.3 & Baimian2 \\
\hline & $q H F F B N-25$ & I & C25(D6) & 69.1 & DPL0702 & 2.82 & 2.83 & -0.49 & -0.59 & 1.22 & $\mathrm{D}$ & 6.2 & Baimian2 \\
\hline & $q H F F B N-9$ & II & C9(A9) & 54.4 & NAU6177 & $3.76^{*}$ & 2.69 & 0.97 & -0.19 & -0.20 & A & 13.9 & CIR12 \\
\hline & $q H F F B N-17$ & II & C17(D3) & 15.9 & NAU2649 & 2.33 & 2.69 & -0.59 & 0.12 & -0.21 & A & 5.2 & Baimian2 \\
\hline & $q H F F B N-L G 3$ & II & LG3 & 6.1 & CGR5576 & 2.17 & 2.69 & -0.47 & -1.31 & 2.77 & D & 11.1 & Baimian2 \\
\hline
\end{tabular}

\footnotetext{
* LOD score is not only $>3$ but also exceeds permutation threshold.

${ }^{a} \mathrm{~A}=$ additive effect.

${ }^{b} \mathrm{D}=$ dominant effect.

${ }^{c} R^{2}=$ phenotypic variation explained.

${ }^{d}$ Favorable gene means the parent provides gene facilitating early maturity.

See Table 1 for abbreviations.
} 
Pop I

C17(D3)

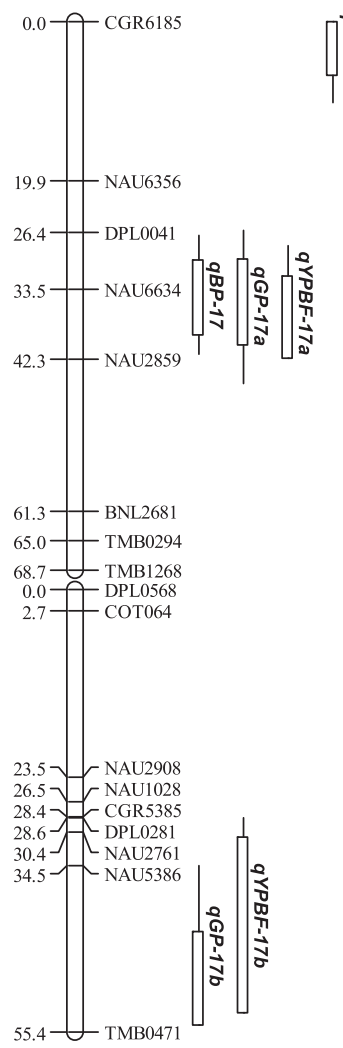

Pop II

C17(D3)
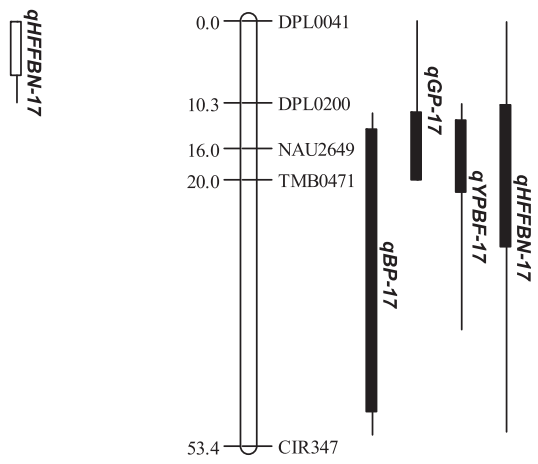

Fig. 3. Location of common QTL in the two populations: Pop I (Baimian $2 \times$ TM-1) and Pop II (Baimian $2 \times$ CIR 12$)$. Four common QTL for four traits are shown as bud period (BP), growth period (GP), yield percentage before frost (YPBF) and height of first fruiting branch node (HFFBN).

for HFFBN were detected in four Chr. (subgenomes) or linkage groups. In Pop I, one suggestive and two significant QTL were detected in C9 (A9), C17 (D3) and C25 (D6), explaining $6.2-13.3 \%$ of the phenotypic variation. The significant QTL $q H F F B N-9$ and $q F F B N-17$ had the LOD scores not only $>3$ but also exceeding permutation threshold. In Pop II, two suggestive and one significant QTL were detected in C9 (A9), C17 (D3) and LG3, explaining 5.2$13.9 \%$ of the phenotypic variation. Here we note that the significant QTL $q H F F B N-9$ had a LOD score not $>3$ but also exceeding permutation threshold. Among all the six QTL, three represented additive effects and three represented dominant or over-dominant effects; decreased height of first fruiting branch node was conferred by Baimian2 allele at five QTL and by CIR12 allele at one QTL.

\section{Common QTL}

It was found from the comparison of QTL across different populations that at least four QTL (significant or suggestive) were common in both populations (Fig. 3). Of these
QTL, $q B P-17$ for bud period (BP), $q G P-17 a / q G P-17 b$ ( $q G P-17$ ) for growth period (GP), $q Y P B F-17 a / q Y P B F-17 b$ $(q Y P B F-17)$ for yield percentage before frost (YPBF) and qHFFBN-17 for height of first fruiting branch node (HFFBN) were always localized close to the bridge markers DPL0041 and/or TMB0471 in C17 in the two populations. Moreover, the favorable genes for these QTL always originated from the same parent (Baimian2), indicating that they are respectively common QTL. In addition, $q Y P B F-12 / 26$ for yield percentage before frost (YPBF) was detected in C12/C26 with the four bridge markers NAU4926, CGR6772, NAU3921 and NAU2170 in both populations. We should note that $q Y P B F-12 / 26$ was tagged at different marker intervals (Figs. 1, 2). Although this pair of QTL have not been confirmed to be common, the favorable genes for them also originated from the same parent (Baimian2) (Table 3), it is supposed to be a common QTL. It is worth noting that $q H F F B N-9$ for height of first fruiting branch node (HFFBN) was always detected close to the bridge marker GH486 in C9 in both populations (Figs. 1, 2). However, the favorable genes for this pair of QTL originated from Baimian2 in Pop I and from CIR12 in Pop II (Table 3), whether they belong to a common QTL requires further experimentation.

\section{Discussion}

Construction of a detailed genetic linkage map is necessary for QTL discovery. However, the low primer polymorphism in intraspecific cotton accessions makes it difficult to expand the number of markers (Li et al. 2008, Iqbal et al. 2001, Wendel et al. 1992). In this study, a total of 4,083 SSR primers were selected from the published interspecific and intraspecific genetic maps in tetraploid cotton (Guo et al. 2007, Nguyen et al. 2004, Qin et al. 2008, Zhang et al. 2012), as well as reported markers linked to QTL for agriculturally significant traits of cotton (Jiang et al. 2009, Lacape et al. 2005, Li et al. 2008, Mei et al. 2004, Qin et al. 2008, Zhang et al. 2003, Zhang et al. 2012). Because the primers derived from the published maps are themselves polymorphic and the markers linked to the genes responsible for agriculturally relevant traits can easily identify genotypic differences among different phenotypes, the polymorphic primer rates of the two populations in this study were relatively higher and respectively reached $7.20 \%$ and $4.14 \%$ by calculation. This feature is useful for the construction of a high-density genetic map and QTL detection.

With regard to the distribution of QTL in A and D subgenomes, Zhang et al. (2008) concluded that more QTL for growing period, yield and fiber quality were distributed in $\mathrm{D}$ subgenome than in A subgenome. However, Lin et al. (2005) and Shen et al. (2005) reported that more QTL for fiber quality were located in A subgenome than in D subgenome. In this study, more QTL for early-maturing traits were stably distributed in D subgenome (16) than in A subgenome (12) in Pop I, whereas more QTL were stably 
distributed in A subgenome (9) than in D subgenome (5) in Pop II. The differences in the distribution of QTL in A and D subgenomes might be associated with the cultivars employed, the number of marker, and the particular QTL identified for different traits. Therefore, whether the agronomically useful genes are more frequently distributed in A or D subgenome should be established in subsequent studies.

Classical genetics has promulgated the opinion that the genetic action pattern of most early-maturing traits corresponds to an additive dominant model. Baker et al. (1973) showed that the dominant variance of early-maturing traits was greater than the additive variance, and the genetic effects of the genes affecting early-maturing traits were primarily dominant. Godoy and Palomo (1999) reported that of 13 early maturity components, all traits, with the exception of days to first square, vertical flowering interval, boll maturation period and production rate index showed significant additive variance. Song et al. (2005) described that four early-maturing traits, namely growth period, date of first flower, boll period and first fruiting branch node exhibited primarily additive effects. In this study, we analyzed the genetic effects of early-maturing traits utilizing a molecular diagnostic and found more QTL displayed dominant or over-dominant effects in both populations. In Pop I, a total of 15 QTL displayed additive effects and 18 QTL displayed dominant or over-dominant effects; in Pop II, a total of 9 QTL displayed additive effects and 12 QTL displayed dominant or over-dominant effects (Table 3). Fan et al. (2006a) identified 10 QTL for early-maturing traits, including first fruiting branch node, bud period, flower and boll period, growth period and yield percentage before frost, of which four QTL displayed additive effects and six displayed dominant or over-dominant effects. This result is consistent with the present findings, suggesting that early-maturing traits may primarily be controlled by dominant and overdominant effects. Of course, owing to the influence of limited markers and QTL analysis software, it is likely that some micro-effect QTL have yet to be detected, limiting our ability to fully explain the inheritance of quantitative traits at the molecular level. This necessitates that classical quantitative and molecular quantitative genetics should be used in tandem in the future to classify the inheritance of earlymaturing traits more accurately.

Limited by QTL reliability, only a minority of QTL identified have been applied to marker-assisted selection in cotton at present (Guo et al. 2005, Wang et al. 2007), and the majority of QTL are still unavailable for breeding. This requires accumulating robust enough data to find common QTL, which have high reliability, can be detected through multiple generations, environments or populations. Sun et al. (2012) carried out QTL mapping for fiber quality traits across multiple generations and environments in upland cotton. They identified nine common QTL for fiber quality traits in $\mathrm{F}_{2}, \mathrm{~F}_{2: 3}$ and RILs simultaneously, of which two QTL for fiber strength were detected in all three generations and all four environments. Qin et al. (2009) also detected eight common QTL for four fiber quality traits across two populations in upland cotton. The above common QTL could be used in marker-assisted selection to improve fiber quality. In this study, at least four common QTL, $q B P-17$ for bud period (BP), $q G P-17 a / q G P-17 b$ ( $q G P-17)$ for growth period (GP), $q Y P B F-17 a / q Y P B F-17 b(q Y P B F-17)$ for yield percentage before frost (YPBF) and $q H F F B N-17$ for height of first fruiting branch node (HFFBN), were detected in both populations. They should be reliable and could be utilized for marker-assisted selection to facilitate cotton early maturity. It was encouraging to note that the common QTL $q B P$ 17 had a LOD score not only $>3$ but also exceeding permutation threshold, explaining $12.6 \%$ of the phenotypic variation (Table 3). This QTL should be considered preferentially in MAS. In addition, $q F F B N-9$ (Pop I) for first fruiting branch node (FFBN), $q F B P-7$ (Pop II) and $q F B P-17$ (Pop I and Pop II) for flower and boll period (FBP) were localized in the same chromosomes of the genetic map constructed by Zhang et al. (2008, 2009). Due to the lack of bridge markers, these QTL may be confirmed as common in future studies through accumulating more information. It was also established in this study that despite the fact that few common QTL for several traits were detected, a strong genetic correlation existed among early-maturing traits (Table 2), notably, seedling period was significant or highly significant positively correlated with bud period, flower and boll period and growth period and significant or highly significant negative correlated with yield percentage before frost. This indicates that utilizing the markers linked closely to minor reliable QTL for individual traits for marker assisted selection can provide an effective approach toward simultaneous improvement of related traits.

\section{Acknowledgements}

This work was supported by grants from High-tech Program 863 (2012AA101108), Achievements Transformation Project of National Agricultural Science and Technology (2010276), Research and Establishment of Modern Industrial Technology System for National Cotton (nycytx-06-09) and Key Scientific Research Project of Henan Province (092102110025, 112102110105).

\section{Literature Cited}

Ai,N.J., X.X.Zhu, R.Z.Guan, J.J.Zhao and T.Z.Zhang (2010) Genetic analysis of major locus group constitutions of growth stages in upland cotton. Sci. Agri. Sin. 43: 4140-4148.

Baker,J.L (1973) The inheritance of several agronomic and fiber properties among selected lines of upland cotton (Gossypium hirsutum L.). Crop Sci. 13: 444-450.

Churchill,G.A. and R.W.Doerge (1994) Empirical threshold values for quantitative trait mapping. Genetics 138: 963-971.

Dong,N., C.Q.Li, Q.L.Wang, N.J.Ai, G.H.Hu and J.B.Zhang (2010) Mixed inheritance of earliness and its related traits of short-season cotton under different ecological environments. Cotton Sci. 22: 304-311. 
Fan,S.L., S.X.Yu, M.Z.Song and R.H.Yuan (2006a) Construction of molecular linkage map and QTL mapping for earliness in shortseason cotton. Cotton Sci. 18: 135-139.

Fan, S.L., S.X.Yu, R.H.Yuan and M.Z.Song (2006b) Genetic effects and environmental interactions of early maturity in short-season cotton. Acta Botanica Boreale-Occidentalia Sin. 26: 2270-2275.

Godoy,A.S. and G.A.Palomo (1999) Genetic analysis of earliness in upland cotton (Gossypium hirsutum L.). I. Morphological and phenological variables. Euphytica 105: 155-160.

Guo,W.Z., T.Z.Zhang, Y.Z.Ding, Y.C.Zhu, X.L.Shen and X.F.Zhu (2005) Molecular marker assisted selection and pyramiding of two QTL for fiber strength in upland cotton. Acta Genet. Sin. 32: $1275-1285$.

Guo, W.Z., C.P.Cai, C.B.Wang, Z.G.Han, X.L.Song, K.Wang, X.W. Niu, C.Wang, K.Y.Lu, B.Shi et al. (2007) A microsatellite-based, gene-rich linkage map reveals genome structure, function, and evolution in Gossypium. Genetics 176: 527-541.

Guo,Y.F., J.C.McCarty, J.N.Jenkins and S.Saha (2008) QTL for node of first fruiting branch in a cross of an upland cotton, Gossypium hirsutum L., cultivar with primitive accession Texas 701. Euphytica 163: 113-122.

Han,Z.G., C.B.Wang, X.L.Song, W.Z.Guo, J.Y.Guo, C.H.Li, X.Y. Chen and T.Z.Zhang (2006) Characteristics, development and mapping of Gossypium hirsutum derived EST-SSRs in allotetraploid cotton. Theor. Appl. Genet. 112: 430-439.

Iqbal,M.J., O.U.K.Reddy and K.M.El-Zik (2001) A genetic bottleneck in the 'evolution under domestication' of upland cotton Gossypium hirsutum L. examined using DNA fingerprinting. Theor. Appl. Genet. 103: 547-554.

Jiang,F., J.Zhao, L.Zhou, W.Z.Guo and T.Z.Zhang (2009) Molecular mapping of Verticillium wilt resistance QTL clustered on chromosomes D7 and D9 in upland cotton. Sci. China, Ser. C: Life Sci. 52: 872-884.

Jiang, C.X., R.J.Wright, K.M.El-Zik and A.H.Paterson (1998) Polyploid formation created unique avenues for response to selection in Gossypium (cotton). Proc. Natl. Acad. Sci. USA 95: 4419-4424.

Kohel,R.J., T.R.Richmond and C.F.Lewis (1970) Texas marker-1. Description of a genetic standard for Gossypium hirsutum L. Crop Sci. 10: 670-671.

Lacape, J.M., T.B.Nguyen, B.Courtois, J.L.Belot, M. Giband, J.P. Gourlot, G.Gawryziak, S. Roques and B.Hau (2005) QTL analysis of cotton fiber quality using multiple $G$. hirsutum $\times G$. barbadense backcross generations. Crop Sci. 45: 123-140.

Lacape, J.M., J.Jacobs，T. Arioli， R. Derijcker， C.N. Forestier，D. Llewellyn, J.Jean, E. Thomas and C.Viot (2009) A new interspecific, Gossypium hirsutum $\times$ G. barbadense, RIL population: towards a unified consensus linkage map of tetraploid cotton. Theor. Appl. Genet. 119: 281-292.

Lander,E.S. and L.Kruglyak (1995) Genetic dissection of complex traits guidelines for interpreting and reporting linkage results. Nat. Genet. 11: 241-247.

Li,C.Q., W.Z.Guo, X.L.Ma and T.Z.Zhang (2008) Tagging and mapping of QTL for yield and its components in upland cotton (Gossypium hirsutum L.) population with varied lint percentage. Cotton Sci. 20: 163-169.

Li,C.Q., Y.Q.Li, Q.L.Wang, N.Dong and J.B.Zhang (2011) Quantitative study of growth period and yield traits in upland cotton (G. hirsutum L.) under different ecological environments. Acta Agri. Boreali-sinica. 26: 140-145.

Lin,Z.X., D.H.He, X.L.Zhang, Y.C.Nie, X.P.Guo, C.D.Feng and M.D.Stewart (2005) Linkage map construction and mapping QTL for cotton fiber quality using SRAP, SSR and RAPD. Plant Breed. 124: $180-187$.

McCouch,S.R., Y.G.Cho, M.Yano, E.Paul, M.Blinstrub, H.Morishima and T.Kinoshita (1997) Report on QTL nomenclature. Rice Genet. Newsl. 14: 11-13.

Mei,M., N.H.Syed, W.Gao, P.M.Thaxton, C.W.Smith, D.M.Stelly and Z.J.Chen (2004) Genetic mapping and QTL analysis of fiberrelated traits in cotton (Gossypium L.). Theor. Appl. Genet. 108: 280-291.

Nguyen,T.B., M.Giband, P.Brottier, A.M.Risterucci and J.M.Lacape (2004) Wide coverage of tetraploid cotton genome using newly developed microsatellite markers. Theor. Appl. Genet. 109: 167175

Paterson,A.H., C.L.Brubaker and J.F.Wendel (1993) A rapid method for extraction of cotton (Gossypium spp.) genomic DNA suitable for RFLP and PCR analysis. Plant Mol. Biol. Rep. 11: 112-127.

Paterson, A.H., Y.Saranga, M.Menz, C.Jiang and R.J.Wright (2003) QTL analysis of genotype $\times$ environment interactions affecting cotton fiber quality. Theor. Appl. Genet. 106: 384-396.

Qin,H.D., W.Z.Guo, Y.M.Zhang and T.Z.Zhang (2008) QTL mapping of yield and fiber traits based on a four-way cross population in Gossypium hirsutum L. Theor. Appl. Genet. 117: 883-894.

Qin, Y.S., R.Z.Liu, H.X.Mei, T.Z.Zhang and W.Z.Guo (2009) QTL mapping for yield traits in upland cotton (Gossypium hirsutum L.). Acta Agro. Sin. 35: 1812-1821.

Reinisch,A.J., J.M.Dong, C.L.Brubaker, D.M.Stelly, J.F.Wendel and A.H.Paterson (1994) A detailed RFLP map of cotton, Gossypium hirsutum $\times$ Gossypium barbadense: chromosome organization and evolution in a disomic polyploidy genome. Genetics 138: 829-847.

Shappley,Z.W., J.N.Jenkins, J.Zhu and J.C.McCarty Jr (1998) Quantitative trait loci associated with agronomic and fiber traits of upland cotton. J. Cotton Sci. 2: 153-163.

Shen,X.L., W.Z.Guo, J.Yu, R.J.Kohel and T.Z.Zhang (2005) Molecular mapping of QTL for fiber qualities in three diverse lines in upland cotton using SSR markers. Mol. Breed. 15: 169-181.

Song,M.Z., S.X.Yu and S.L.Fan (2005) Genetic analysis of main agronomic traits in short season upland cotton. Cotton Sci. 17: 94-98.

Sun,F.D., J.H.Zhang, S.F.Wang, W.K.Gong, Y.Z.Shi, A.Y.Liu, J.W. Li, J.W.Gong, H.H.Shang and Y.L.Yuan. (2012) QTL mapping for fiber quality traits across multiple generations and environments in upland cotton. Mol. Breed. 30: 569-582.

Tan,L.W. and Z.D.Liu (1990) Research on selection and varietal traits of Zhongmian 12. Sci. Agric. Sin. 23: 12-19.

Ulloa, M., W.R.Meredith, Z.W. Shappley and A.L.Kahler (2002) RFLP genetic linkage maps from four $\mathrm{F} 2: 3$ populations and a joinmap of Gossypium hirsutum L. Theor. Appl. Genet. 104: 200-208.

Van Ooijen, J.W. and R.E.Voorrips (2001) JoinMap ${ }^{R}$ Version 3.0: software for the calculation of genetic linkage maps.CPRO-DLO, Wageningen.

Voorrips,R.E. (2006) MapChart 2.2: software for the graphical presentation of linkage maps and QTLs. Plant Research International, Wageningen.

Wang,F.R., R.Z.Liu, L.M.Wang, C.Y.Zhang, G.D.Liu, Q.H.Li., X.B. Ma and J.Zhang (2007) Molecular markers of Verticillium wilt resistance in upland cotton (Gossypium hirsutum L.) cultivar and their effects on assisted phenotypic selection. Cotton Sci. 19: 424430.

Wang, S., C.J.Basten and Z.B.Zeng (2006) Windows QTL Cartographer 2.5. Department of Statistics, North Carolina State University, Raleigh.

Wendel,J.F., C.L.Brubaker and E.Percival (1992) Genetic diversity in 
Gossypium hirsutum and the origin of upland cotton. Am. J. Bot. 79: 1291-1310.

White,T.G. (1966) Diallel analysis of quantitatively inherited characters in Gossypium hirsutum L. Crop Sci. 6: 253-295.

$\mathrm{Yu}$,J., R.J.Kohel and C.W.Smith (2010) The construction of a tetraploid cotton genome wide comprehensive reference map. Genomics 95: 230-240.

Yu,S.X. and Z.M.Huang (1990) Inheritance analysis on earliness components of short season cotton varieties in G. hirsutum. Sci. Agri. Sin. 23: 48-54.

Zeng,Z.B (1994) Precision mapping of quantitative trait loci. Genetics 136: $1457-1468$.

Zhang,J., W.Z.Guo and T.Z.Zhang (2002) Molecular linkage map of allotetraploid cotton (Gossypium hirsutum L. $\times$ Gossypium barbadense L.) with a haploid population. Theor. Appl. Genet. 105: 1166-1174.

Zhang,K., J.Zhang, J.Ma, S.Y.Tang, D.J.Liu, Z.H.Teng, D.X.Liu and Z.S.Zhang (2012) Genetic mapping and quantitative trait locus analysis of fiber quality traits using a three-parent composite popu- lation in upland cotton (Gossypium hirsutum L). Mol. Breed. 29: 335-348.

Zhang,T.Z., Y.L.Yuan, J.Yu, W.Z.Guo and R.J.Kohel (2003) Molecular tagging of a major QTL for fiber strength in upland cotton and its marker-assisted selection. Theor. Appl. Genet. 106: 262-268.

Zhang,X.L., K.B.Wang, G.L.Song, F.Liu, S.H.Li, C.Y.Wang, X.D. Zhang and Y.H.Wang (2008) Primary QTL mapping of upland cotton RIL CRI-G6 by SSR marker. Cotton Sci. 20: 192-197.

Zhang, X.L., J.S.Gao, G.L.Song, F.Liu, S.H.Li, K.F.Liu, Z.Y.Chu, Y.Y.Shao and K.B.Wang (2009) Additive and epistatic effects QTL analysis on upland cotton CRI-G6. Mol. Plant Breed. 2: 312320 .

Zhang,Z.S., M.C.Hu, J.Zhang, D.J.Liu, J.Zheng, K.Zhang, W.Wang and Q.Wan (2009) Construction of a comprehensive PCR-based marker linkage map and QTL mapping for fiber quality traits in upland cotton (Gossypium hirsutum L.). Mol. Breed. 24: 49-61.

Zhu, G.L., C.H.Wang, X.H.Guo, W.K.Gao and Y.Y.Gan (2008) The preliminary research on the growth characteristics of Baimian2. Henan Agri. Sci. 15: 47-50. 